\title{
Influence of hydrodynamic induced cavitation on water pollutants
}

\author{
A. Schmid \\ University of Applied Sciences, Hof, Germany
}

\begin{abstract}
Hydrodynamic induced cavitation generates imploding cavitation bubbles which can lead to degradation or even mineralisation of water constituents without the addition of any chemicals. This technology overcomes the restrictions of ultrasound irradiation by the local production of a cavitation cloud close to the sonotrodes. In the presented paper hydrodynamic cavitation can be stabilized and extended inside a pipe downstream the nozzle depending on the ambient pressure conditions. If the pressure is kept low, the imploding cavitation bubbles generate new cavities, analogous to a chain reaction, and elevate the radical synthesis inside the apparatus. During the pilot tests MTBE and ETBE were degraded and complete mineralisation started at a time delay of approximately 20 minutes. Expanding the experiments to ethan-1,2-diol, the biological degradation characteristics change significantly depending on cavitation exposure time. A significant difference on chemical effects was observed between imploding cavitation bubbles and supercavitation. Supercavitation can mobilize chemical stable compounds for further treatment.
\end{abstract}

Keywords: hydrodynamic cavitation, supercavitation, biological degradation, MTBE, ETBE, ethan-1,2-diol.

\section{Introduction}

MTBE (Methyl tert-butyl ether), ETBE (Ethyl-tert-butylether) and other chemical contaminants can be degraded under ultrasonic irradiation (Kang and Hoffmann [1], Hoffmann et al. [2], Nam et al. [3]) by reactions with several $\mathrm{OH} \bullet$-radicals, created from imploding cavitation bubbles. Using ultrasound, a cloud of cavitation bubbles is generated close to the sonotrodes, limiting the overall efficiency of the reactor (Horst and Hoffmann [4]). To overcome these problems, hydrodynamic 
induced cavitation in combination with high turbulences may improve the elimination efficiency of the water contaminants.

Ethan-1,2-diol is commonly used as a de-icing-agent of airplanes and railways and should be treated to meet environmental standards (Rebsdat and Mayer [5]). At present, these de-icing-agents act as dilution components to other wastewater streams, leading to lower concentrations.

\section{Material and methods}

The cavitational flow regime is created by a nozzle downstream a motor-pump and is kept up inside a pipe with reduced diameter over a length of 2 meters following the cavitation-nozzle. To maintain cavitation downstream the nozzle, low ambient pressure conditions must be created to enable repeated cavitation bubble development, analogous to a chain reaction (Geisler [6]). When cavitation bubbles implode, the fluid close to the bubble will accelerate and as a consequence the local pressure declines, till evaporation pressure is reached and new cavities are created again. This is strongly dependent on the ambient pressure conditions and the specific energy input for cavitation induction (Schmid [7]).

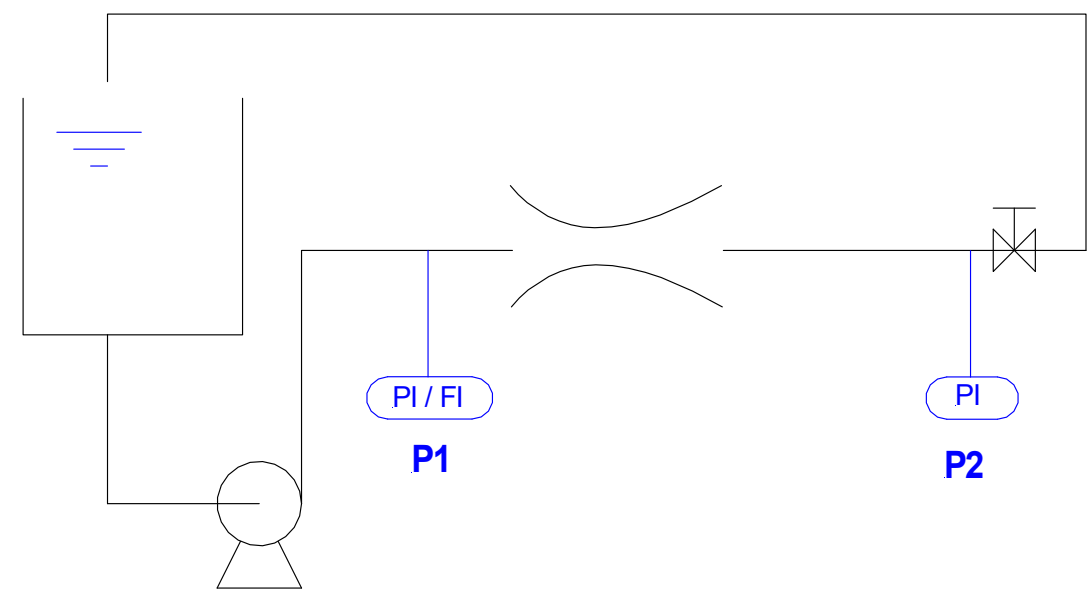

Figure 1: Pilot plant.

\section{Results}

Depending on the specific energy input and variations of the pressure $\mathrm{p}_{2}, \mathrm{MTBE}$ and ETBE can be degraded inside a cavitational flow regime without addition of any chemicals (see Figure 2).

Mineralisation starts with a time delay of app. 20 minutes (see Figure 3) when the COD-concentrations declines. This implies that ETBE is oxidised in a multistep reaction by hydrodynamic induced cavitation, finally leading to a complete oxidation of the water contaminant to $\mathrm{CO}_{2}$ and $\mathrm{H}_{2} \mathrm{O}$. 


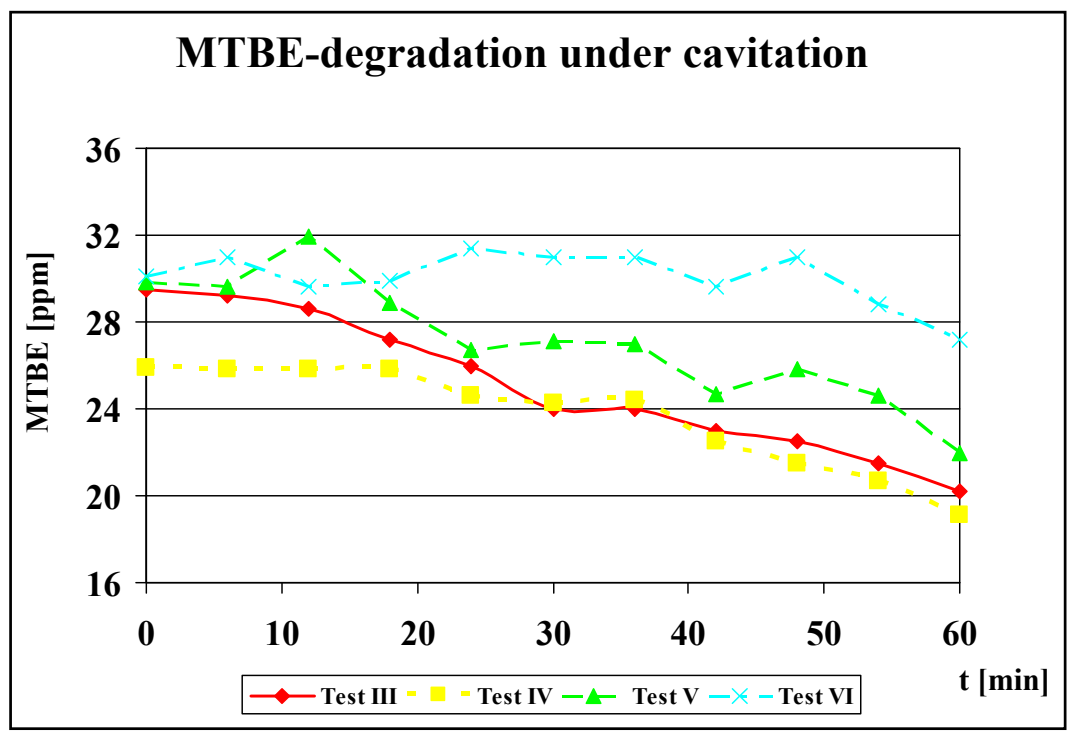

Figure 2: MTBE-degradation with pilot plant.

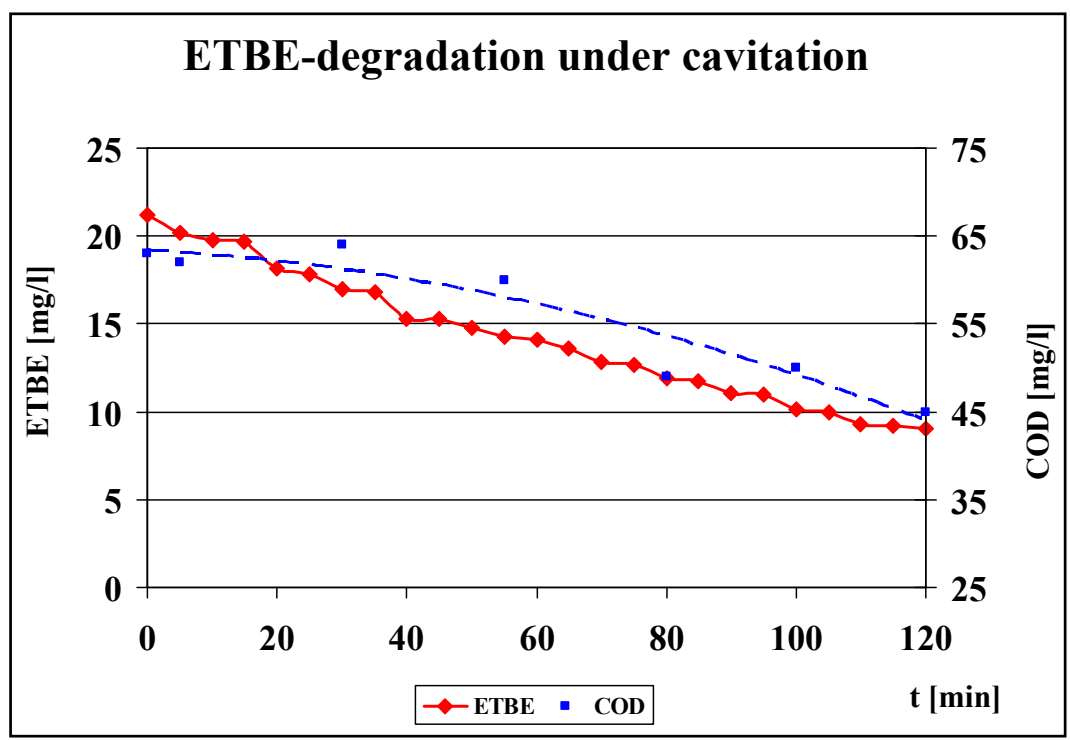

Figure 3: ETBE-degradation with pilot plant.

Taking ethan-1,2-diol, the biological degradation characteristics changes significantly depending on the exposed time of imploding cavitation bubbles/supercavitation (see Figures 4 and 5). 


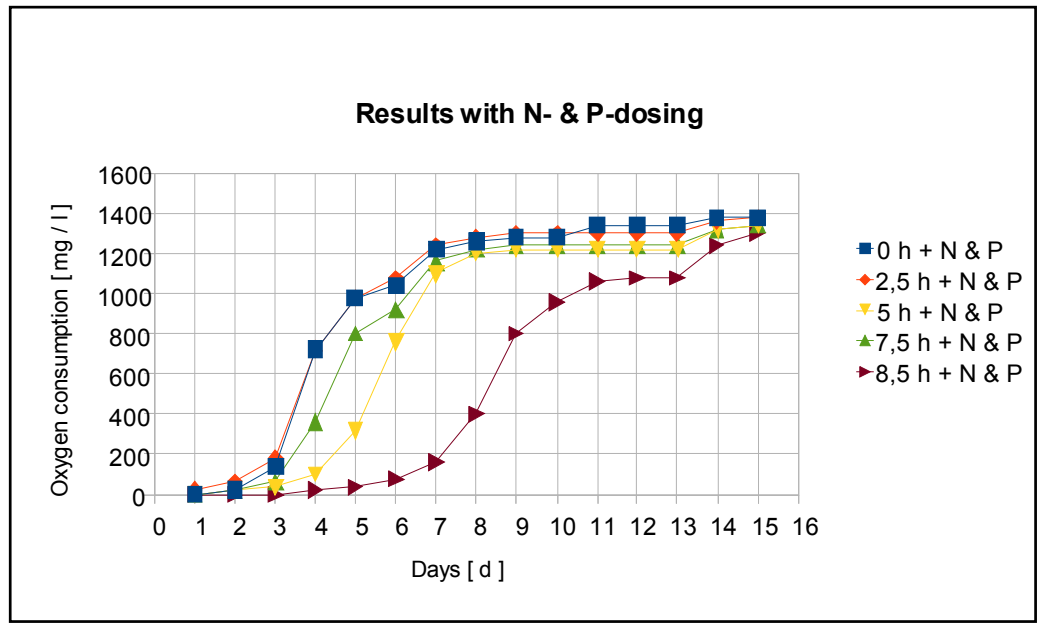

Figure 4: Changes of BOD at different durations of cavitation exposure (imploding cavitation).

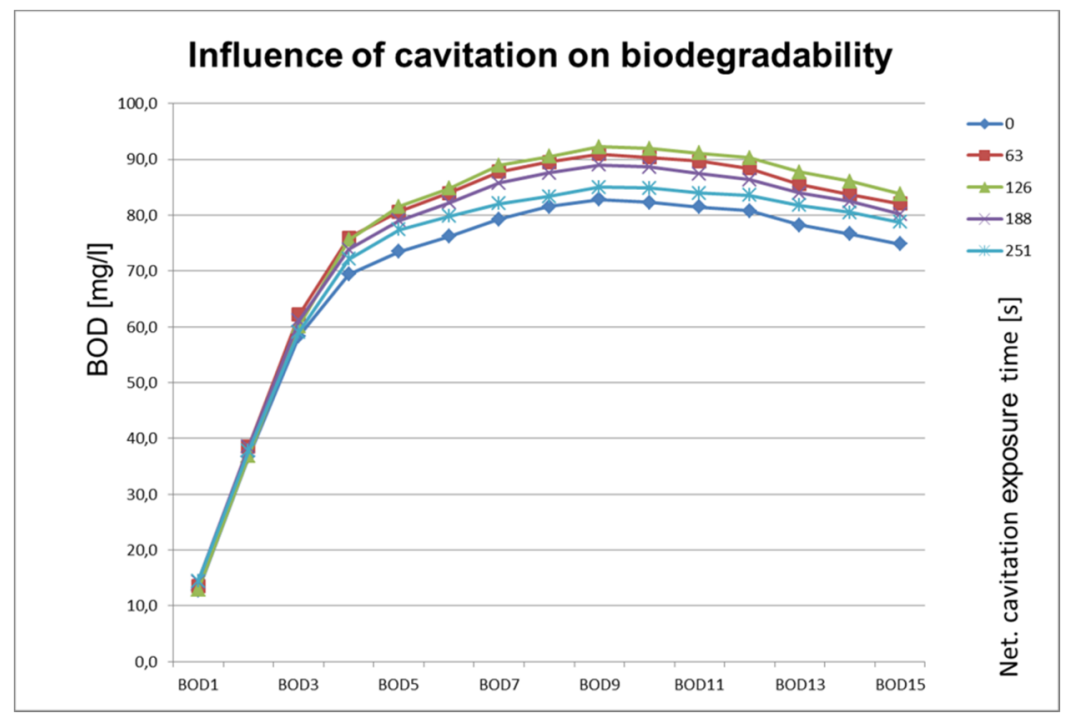

Figure 5: Changes of BOD by imposure of supercavitation.

An optimum was found, increasing the biodegradability by app. $15 \%$ after a net supercavitation exposure time of around 1.5 minutes (see Figure 5).

Increasing the cavitation exposure time, using supercavitation (70 cm length), the deicing-agent obviously released new COD-load (see Figure 6), leading to a significant reduction of the $\mathrm{BOD}_{5} / \mathrm{COD}$-ratio (see Figure 7 ), which can't be observed when imploding cavitation bubbles interfere with the chemicals. 
Hydrodynamic cavitation, especially supercavitation, is capable to mobilize unknown stable chemical compounds to improve chemical and biochemical degradation of hardly degradable substances.

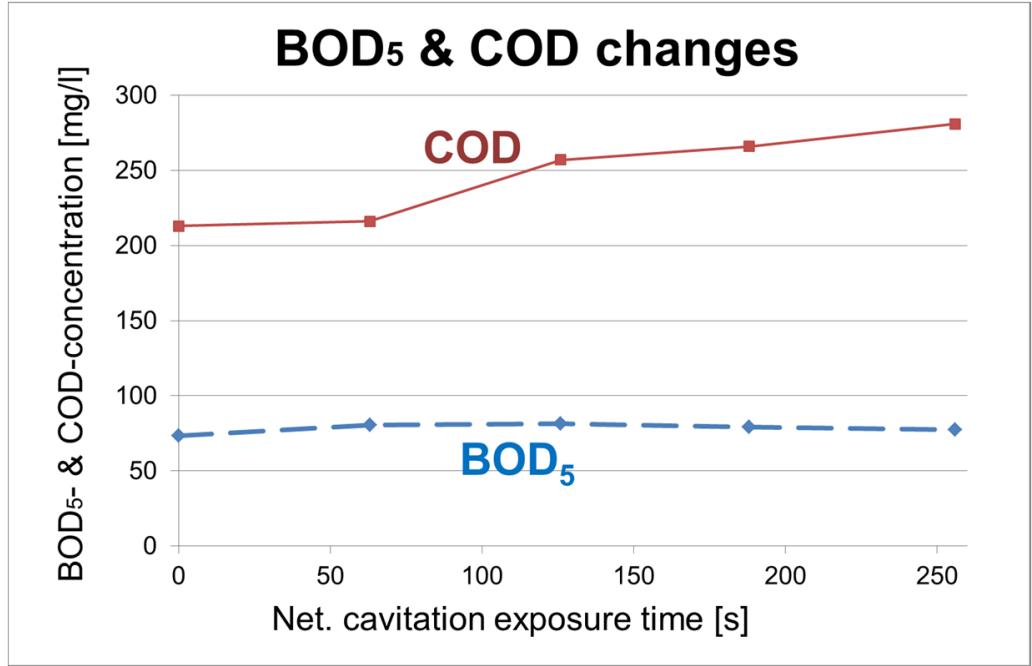

Figure 6: Changes of $\mathrm{BOD}_{5}$ and COD under supercavitation.

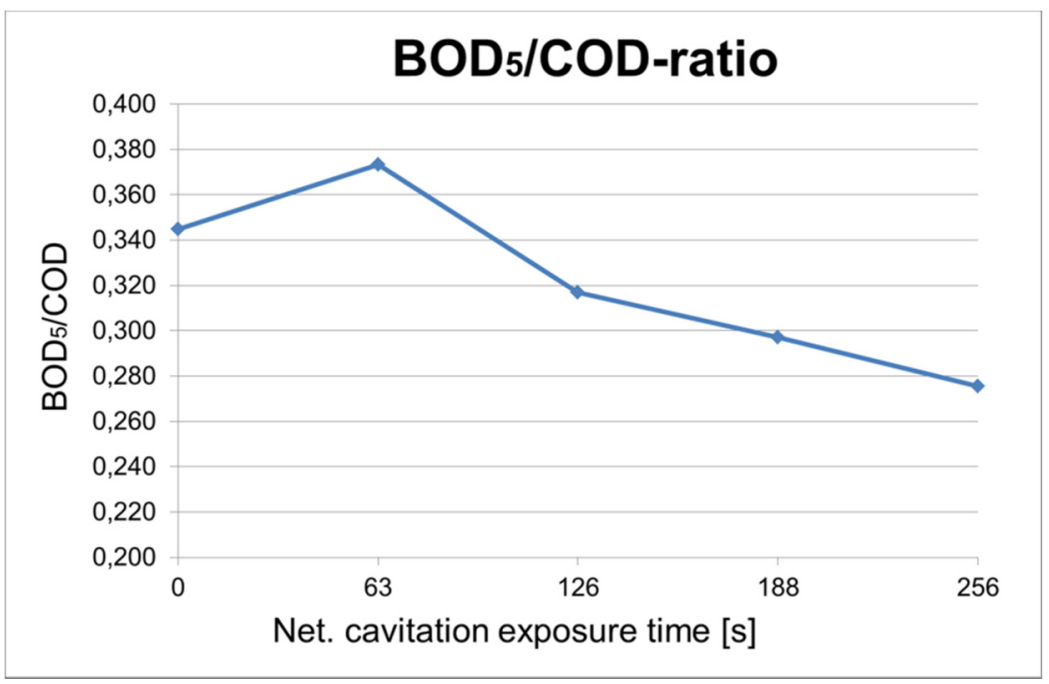

Figure 7: $\quad \mathrm{BOD}_{5} / \mathrm{COD}$-ratio in dependence on supercavitation exposure. 


\section{Conclusion and discussion}

Hydrodynamic induced cavitation is capable to modify diverse chemicals, improve chemical as well as biochemical degradation characteristics and may even be used for complete mineralization without addition of any chemicals. The unknown intermediates originated from ethan-1,2-diol can be degraded under aerobic conditions, leading to a $15 \%$ additional oxygen requirement after supercavitation exposure of app. 1.5 minutes. After 1 minute COD increases, indicating that stable chemical compounds are transferred toward reactive compounds.

The presented study shows an easy technology to modify water by cavitation exposure to form radicals, which are capable to increase elimination rates and change chemical structures of different trace pollutants. The system was run with a commercial centrifugal pump, generating a pressure of app. 3.5 bar at a flow rate of app. $5 \mathrm{l} / \mathrm{s}$.

The chemical modifications are strongly dependent on the form of cavitation imploding cavitation bubbles or supercavitation. Supercavitation can obviously mobilize chemically stable compounds, which might be treated in a downstream biological reactor. Much more research is needed to work out optimum degradation rates in dependence on energy input and different types of cavitation.

This technology is not restricted to the investigated chemicals only; it is very likely, that this technology can be applied to other interfering water constituents, too. Main focus should concentrate on changes on biodegradability for final elimination inside biological reactors to reduce energy consumption, instead of complete mineralisation.

\section{References}

[1] Kang, J., Hoffmann, M., Kinetics and mechanism of the sonolytic destruction of methyl tert-butyl ether by ultrasonic irradiation in the presence of ozone. Environmental Science \& Technology, 32, 3194-3199, 1998.

[2] Hoffmann M, Hua I., Höchemer R., Application of ultrasonic irradiation for the degradation of chemical contaminants in water. Ultrasonics Sonochemistry, 3, 163-172, 1996.

[3] Nam S., Han S., Kang J., Choi H., Kinetics and mechanisms of the sonolytic destruction of non-volatile organic compounds: Investigation of the sonochemical reaction zone using $\mathrm{OH} \cdot$ monitoring techniques. Ultrasonics Sonochemistry, 10, 139-147, 2003.

[4] Horst C., Hoffmann U., Design, operation and characterization of ultrasound reactors. From: Tiehm A., Neis U., Ultrasound in Environmental Engineering. TU Hamburg-Harburg Reports on Sanitary Engineering, 25, 77-90, 1999.

[5] Rebsdat S., Mayer D., Ethylene Glycol, Ullmann's Encyclopedia of Industrial Chemistry, Weinheim, Wiley-VCH, 2005. 
[6] Geisler R., Untersuchungen zur laserinduzierten Kavitation mit Nanosekunden- und Femtosekundenlasern. University Göttingen, 2003.

[7] Schmid A., Verfahren und Vorrichtung zur Behandlung von schadstoffhaltigem Wasser. German Patent DE 102004009 636, 2004. 